\title{
A SIMPLE PROOF OF JACOBI'S FOUR-SQUARE THEOREM
}

\author{
M. D. HIRSCHHORN
}

(Communicated by Larry J. Goldstein)

\begin{abstract}
Jacobi's four-square theorem, which gives the number of representations of a positive integer as a sum of four squares, is shown to follow simply from the triple-product identity.
\end{abstract}

1. Recently [2], I showed how one can obtain Jacobi's two-square theorem from the triple-product identity. In this note I show that the triple-product identity also gives Jacobi's four-square theorem:

THEOREM 1. The number $r_{4}(n)$ of representations of the positive integer $n$ as a sum of four squares is given by

$$
r_{4}(n)=8 \sum_{d \mid n, 4+d} d
$$

2. The triple product identity is

$$
\prod_{n \geqslant 1}\left(1+a x^{2 n-1}\right)\left(1+a^{-1} x^{2 n-1}\right)\left(1-x^{2 n}\right)=\sum_{-\infty}^{\infty} a^{n} x^{n^{2}} .
$$

It follows easily (loc. cit.) that

(1) $\left(a-a^{-1}\right) \prod_{n \geqslant 1}\left(1-a^{2} x^{n}\right)\left(1-a^{-2} x^{n}\right)\left(1-x^{n}\right)=\sum_{-\infty}^{\infty}(-1)^{n} a^{2 n+1} x^{\left(n^{2}+n\right) / 2}$.

Differentiate (1) with respect to $a$, put $a=1$, divide by 2 and we obtain the identity

$$
\prod_{n \geqslant 1}\left(1-x^{n}\right)^{3}=\frac{1}{2} \sum_{-\infty}^{\infty}(-1)^{n}(2 n+1) x^{\left(n^{2}+n\right) / 2} .
$$

This is a celebrated identity of Jacobi [1, Theorem 357], and can be considered our starting point.

Squaring (2) gives

$$
\prod_{n \geqslant 1}\left(1-x^{n}\right)^{6}=\frac{1}{4} \sum_{m, n=-\infty}^{\infty}(-1)^{m+n}(2 m+1)(2 n+1) x^{\left(m^{2}+n^{2}+m+n\right) / 2} .
$$

Received by the editors April 15, 1986 and, in revised form, July 15, 1986.

1980 Mathematics Subject Classification (1985 Revision). Primary 11E25, 11 P05.

C1987 American Mathematical Society $0002-9939 / 87 \$ 1.00+\$ .25$ per page 
Now split the sum on the right into two, according as $m+n$ is even or odd, to obtain

$$
\begin{aligned}
\prod_{n \geqslant 1}\left(1-x^{n}\right)^{6}=\frac{1}{4}\left\{\sum_{m \equiv n(\bmod 2)}(2 m+1)(2 n+1) x^{\left(m^{2}+n^{2}+m+n\right) / 2}\right. & \\
& \left.-\sum_{\substack{m \neq n(\bmod 2)\\
}}(2 m+1)(2 n+1) x^{\left(m^{2}+n^{2}+m+n\right) / 2}\right\} .
\end{aligned}
$$

In the first sum, set $r=\frac{1}{2}(m+n), s=\frac{1}{2}(m-n)$, and in the second set $r=$ $\frac{1}{2}(m-n-1), s=\frac{1}{2}(m+n+1)$, and, remarkably, the two sums coalesce to give

$$
\prod_{n \geqslant 1}\left(1-x^{n}\right)^{6}=\frac{1}{2} \sum_{r, s=-\infty}^{\infty}\left((2 r+1)^{2}-(2 s)^{2}\right) x^{r^{2}+s^{2}+r} \text {. }
$$

Once again splitting the sum, we obtain

$$
\begin{array}{r}
\prod_{n \geqslant 1}\left(1-x^{n}\right)^{6}=\frac{1}{2}\left\{\sum_{s=-\infty}^{\infty} x^{s^{2}} \sum_{r=-\infty}^{\infty}(2 r+1)^{2} x^{r^{2}+r}-\sum_{r=-\infty}^{\infty} x^{r^{2}+r} \sum_{s=-\infty}^{\infty}(2 s)^{2} x^{s^{2}}\right\} \\
=\frac{1}{2}\left\{\sum_{s=-\infty}^{\infty} x^{s^{2}} \times\left(1+4 x \frac{d}{d x}\right) \sum_{r=-\infty}^{\infty} x^{r^{2}+r}-\sum_{r=-\infty}^{\infty} x^{r^{2}+r} \times 4 x \frac{d}{d x} \sum_{s=-\infty}^{\infty} x^{s^{2}}\right\} .
\end{array}
$$

Making use of the triple-product identity, we obtain

$$
\begin{aligned}
\prod_{n \geqslant 1}\left(1-x^{n}\right)^{6}=\frac{1}{2}\{ & \prod_{n \geqslant 1}\left(1+x^{2 n-1}\right)^{2}\left(1-x^{2 n}\right) \\
& \times\left(1+4 x \frac{d}{d x}\right) 2 \prod_{n \geqslant 1}\left(1+x^{2 n}\right)^{2}\left(1-x^{2 n}\right) \\
& -2 \prod_{n \geqslant 1}\left(1+x^{2 n}\right)^{2}\left(1-x^{2 n}\right) \\
& \left.\times 4 x \frac{d}{d x} \prod_{n \geqslant 1}\left(1+x^{2 n-1}\right)^{2}\left(1-x^{2 n}\right)\right\} .
\end{aligned}
$$

Employing the product rule to evaluate the derivatives, we find

$$
\begin{aligned}
\prod_{n \geqslant 1}\left(1-x^{n}\right)^{6}= & \prod_{n \geqslant 1}\left(1+x^{2 n-1}\right)^{2}\left(1-x^{2 n}\right)\left(1+x^{2 n}\right)^{2}\left(1-x^{2 n}\right) \\
& \times\left\{1+8 \sum_{n \geqslant 1} \frac{2 n x^{2 n}}{1+x^{2 n}}-4 \sum_{n \geqslant 1} \frac{2 n x^{2 n}}{1-x^{2 n}}\right\} \\
& -\prod_{n \geqslant 1}\left(1+x^{2 n}\right)^{2}\left(1-x^{2 n}\right)\left(1+x^{2 n-1}\right)^{2}\left(1-x^{2 n}\right) \\
& \times\left\{8 \sum_{n \geqslant 1} \frac{(2 n-1) x^{2 n-1}}{1+x^{2 n-1}}-4 \sum_{n \geqslant 1} \frac{2 n x^{2 n}}{1-x^{2 n}}\right\},
\end{aligned}
$$

or,

$$
\begin{aligned}
\prod_{n \geqslant 1}\left(1-x^{n}\right)^{6}= & \prod_{n \geqslant 1}\left(1+x^{2 n-1}\right)^{2}\left(1+x^{2 n}\right)^{2}\left(1-x^{2 n}\right)^{2} \\
& \times\left\{1-8 \sum_{n \geqslant 1}\left(\frac{(2 n-1) x^{2 n-1}}{1+x^{2 n-1}}-\frac{2 n x^{2 n}}{1+x^{2 n}}\right)\right\}
\end{aligned}
$$


Dividing both sides by

$$
\begin{aligned}
\prod_{n \geqslant 1}\left(1+x^{n}\right)^{4}\left(1-x^{n}\right)^{2} & =\prod_{n \geqslant 1}\left(1+x^{n}\right)^{2}\left(1-x^{2 n}\right)^{2} \\
& =\prod_{n \geqslant 1}\left(1+x^{2 n-1}\right)^{2}\left(1+x^{2 n}\right)^{2}\left(1-x^{2 n}\right)^{2}
\end{aligned}
$$

we obtain

$$
\prod_{n \geqslant 1}\left(\frac{1-x^{n}}{1+x^{n}}\right)^{4}=1-8 \sum_{n \geqslant 1}\left(\frac{(2 n-1) x^{2 n-1}}{1+x^{2 n-1}}-\frac{2 n x^{2 n}}{1+x^{2 n}}\right) .
$$

Now, it is a simple consequence of the triple-product identity that

$$
\prod_{n \geqslant 1}\left(\frac{1-x^{n}}{1+x^{n}}\right)=\sum_{-\infty}^{\infty}(-1)^{n} x^{n^{2}}
$$

so we have

$$
\left(\sum_{-\infty}^{\infty}(-1)^{n} x^{n^{2}}\right)^{4}=1-8 \sum_{n \geqslant 1}\left(\frac{(2 n-1) x^{2 n-1}}{1+x^{2 n-1}}-\frac{2 n x^{2 n}}{1+x^{2 n}}\right) .
$$

Putting $-x$ for $x$, we obtain

$$
\begin{aligned}
\left(\sum_{-\infty}^{\infty} x^{n^{2}}\right)^{4} & =1+8 \sum_{n \geqslant 1}\left(\frac{(2 n-1) x^{2 n-1}}{1-x^{2 n-1}}+\frac{2 n x^{2 n}}{1+x^{2 n}}\right) \\
& =1+8 \sum_{n \geqslant 1}\left(\frac{(2 n-1) x^{2 n-1}}{1-x^{2 n-1}}+\frac{2 n x^{2 n}}{1-x^{2 n}}\right)-8 \sum_{n \geqslant 1}\left(\frac{2 n x^{2 n}}{1-x^{2 n}}-\frac{2 n x^{2 n}}{1+x^{2 n}}\right) \\
& =1+8 \sum_{n \geqslant 1} \frac{n x^{n}}{1-x^{n}}-8 \sum_{n \geqslant 1} \frac{4 n x^{4 n}}{1-x^{4 n}},
\end{aligned}
$$

or,

$$
\left(\sum_{-\infty}^{\infty} x^{n^{2}}\right)^{4}=1+8 \sum_{4 \nmid n} \frac{n x^{n}}{1-x^{n}}
$$

from which Theorem 1 follows directly.

\section{REFERENCES}

1. G. H. Hardy and E. M. Wright, An introduction to the theory of numbers, 4th ed., Clarendon Press, Oxford.

2. Michael D. Hirschhorn, A simple proof of Jacobi's two-square theorem, Amer. Math. Monthly 92 (1985), 579-580.

School of Mathematics, University of New South Wales, P. O. Box 1, Kensington, New South Wales, Australia 2033 